\title{
REGIONAL ANAESTHESIA CAN PLAY AN IMPORTANT ROLE IN MULTIMODAL ANALGESIA FOR MASTECTOMY AND BREAST RECONSTRUCTIVE SURGERY
}

A. Tay ${ }^{1}$, FP. Henry², B. Bellew ${ }^{1}$.

1 St Mary's Hospital- Imperial College Healthcare NHS Trust, Department of Anaesthesia, London, United Kingdom. ${ }^{2}$ Charing Cross Hospital- Imperial College Healthcare NHS Trust, Department of Plastic \& Reconstructive Surgery, London, United Kingdom.

\begin{abstract}
Reconstructive surgery is discussed with the majority of women in the UK undergoing mastectomy either as an immediate or a delayed procedure. Acute postsurgical pain is commonly associated with both mastectomies and reconstructions ${ }^{1}$. Risk of chronic pain is increased with inadequate pain management in the immediate postoperative period ${ }^{2}$. Post-mastectomy pain syndrome is associated with severe pain after mastectomy and adds to an already psychologically devastating experience and negatively impacts the woman's quality of life $^{3}$. The American Society of Anesthesiologists Task Force recommends use of multimodal analgesic strategies including regional blockade 4 .

A 62-year old female presented for mastectomy and immediate reconstruction with a deep inferior epigastric perforator (DIEP) free flap. She had bilateral erector spinae plane (ESP) nerve block catheters sited and rightsided Serratus Anterior block (SAP) prior to TIVA general anaesthesia.
\end{abstract}

\begin{abstract}
Intraoperatively she received standard protocol analgesia. In the post-operative recovery area she was comfortable requiring no further analgesia. Post-operative analgesia included paracetamol, naproxen \& pregabalin. The ESP catheters were bolused at midnight on the day of surgery, 6am and noon the next day with $20 \mathrm{ml} 0.125 \%$ bupivacaine with 1:400,000 adrenaline. Both catheters were removed following final bolus. No rescue analgesia was required until day 2 postoperatively.

ESP nerve block catheters, SAP nerve block, together with multimodal analgesia is a useful post-operative analgesic strategy for mastectomy and DIEP reconstruction. This patient had a $50 \%$ reduction in opiate requirement and we plan to undertake a larger case series using this strategy to refine our center's analgesic protocols for this patient group.
\end{abstract}

References:

1. Amaya F, Hosokawa T, Okamoto A, et al. Can acute pain treatment reduce postsurgical comorbidity after breast cancer surgery? A literature review. Biomed Res Int. 2015;2015:641508.

2. Andersen KG, Duriaud HM, Jensen HE, et al. Predictive factors for the development of persistent pain after breast cancer surgery. Pain. 2015;156:2413-2422.

3. Butz DR, Shenaq DS, Rundell VLM, et al. Postoperative pain and length of stay lowered by use of Exparel in immediate, implant-based breast reconstruction. Plast Reconstr Surg Glob Open. 2015;3:e391.

4. American Society of Anesthesiologists Task Force on Acute Pain Management. Practice guidelines for acute pain management in theperioperative setting: an updated report by the American Society of Anesthesiologists Task Force on Acute Pain Management. Anesthesiology. 2012;116:248-273. 